\title{
Treatment of hypertension: choosing the first-line treatment
}

\author{
Dr. Franz H. Messerli in an interview with Dr. Roman Jaeschke: part 1
}

Franz H. Messerli, IMD, FACC, FACP

Professor at the Division of Cardiology, Icahn School of Medicine, Mount Sinai Health Center, New York, United States; he participated in numerous clinical trials in hypertension and conducted a number of meta-analyses; he received several awards for his contribution to the field; an author or coauthor of more than 750 publications

Professor Messerli, you are the person who led the treatment of hypertension over the last few decades. I would like to ask you to reflect on the last few decades of hypertensive treatment. Where did we start, where are we now?

That is actually a very loaded question and not easy to answer. If you go back to the guidelines, you have to consider that for years and decades the dictum was thiazide diuretics. First it was thiazide diuretics, and then it was low-dose thiazide diuretics. To the practicing physician in the United States this very simply means hydrochlorothiazide. So a few years back I decided to look at the data-what data do we have for hydrochlorothiazide? Surprise, surprise: we have no data for hydrochlorothiazide showing that in a low dose of 12.5 to $25 \mathrm{mg}$ it reduces heart attacks, stroke, or death. So what these guidelines had been telling practicing physicians was all based on either chlorthalidone (INN chlortalidone) or indapamide, yet these two drugs are not used in the US - they are only about $1 \%$ of diuretics used-so clearly what was taught by the guidelines in the past was deceptive.

Now we are realizing finally that we should go with the evidence and we should use chlorthalidone, we should use indapamide, and not hydrochlorothiazide. Unfortunately, those fixed combinations - that is $\beta$-blockers, angiotensin-converting enzyme inhibitors (ACEIs), and angiotensin receptor blockers (ARBs) - are fixed together with hydrochlorothiazide, because pharmaceutical companies were exactly under the same influence. They had the idea hydrochlorothiazide is the way to go. Now we are learning it is not necessarily so.
We should do better. I think by and large chlorthalidone or indapamide have a renaissance.

The other issue to be considered is that we were so convinced that ACEIs and ARBs are the way to go that we almost forgot that calcium channel blockers (CCBs) are as efficacious-if not more efficacious - than ACEIs and ARBs, and we know now that amlodipine and to some extent nifedipine are very well-documented drugs that have excellent outcome data and have been shown to reduce morbidity, mortality, heart attack, stroke, and death, in some cases even better than the ACEIs and ARBs.

In a nutshell, what we do know is that chlorthalidone or indapamide as diuretics, amlodipine and nifedipine as CCBs, and the ARBs and ACEIs are all suitable for first-time therapy. Clearly we have learned that $\beta$-blockers are no longer in that position.

A loaded question but an incredibly informative answer. The notion that hydrochlorothiazide data are almost nonexistent but those for chlorthalidone and indapamide are available is revolutionary for most of the primary practitioners, including myself. The dose of chlorthalidone would be similar to that of hydrochlorothiazide?

Actually no, it is about half. We just recently published a paper in the Journal of the American College of Cardiology ${ }^{1}$ showing that $6.25 \mathrm{mg}$ is a decent antihypertensive dose of chlorthalidone. So you can use a minimal dose. In the US, we actually have the problem that the lowest dose available is $25 \mathrm{mg}$, so if you break the tablet it usually crumbles, but I tell my patients, "Just take a crumb."

McMaster Perspective This interview is part of the McMaster Perspective collection. The video can be viewed at www.mcmtextbook.com. Published with modifications with permission from Dr. Franz H. Messerli. 
How to cite Messerli FH, Jaeschke R. Treatment of hypertension: choosing the first-line treatment. Dr. Franz H. Messerli in an interview with Dr. Roman Jaeschke: part 1. Pol Arch Intern Med. 2017; 127 : 219-220. doi:10.20452/pamw.3992

\section{REFERENCES}

1 Pareek AK, Messerli FH, Chandurkar NB, et al. Efficacy of low-dose chlorthalidone and hydrochlorothiazide as assessed by 24-h ambulatory blood pressure monitoring. J Am Coll Cardiol. 2016; 67: 379-389. doi:10.1016/j.jacc.2015.10.083 